\title{
Chaotic characteristics analysis of simulation signal of ultrasonic nonlinear modulation effect
}

\author{
Hanying Mao ${ }^{1}$, Yuhua Zhang ${ }^{2, a}$, Hanling $\mathrm{Mao}^{3}$ and Zhenfeng Huang ${ }^{3}$ \\ ${ }^{1}$ College of Automobile and Transportation, Guangxi University of Science and Technology, Liuzhou 545006, China \\ ${ }^{2}$ Institute of Light Industrial and Food Engineering, Guangxi University, Nanning 530004, China †Email: \\ ${ }^{3}$ College of Mechanical Engineering, Guangxi University, Nanning 530004, China
}

\begin{abstract}
The effectiveness of chaos and fractal theory in analyzing ultrasonic nonlinear modulation signal was theoretically verified and a new way of using chaos and fractal theory in nondestructive testing is provided based on the simulation signal of ultrasonic nonlinear modulation effect. By constructing the simulation signal of modulation effect, the simulation signal with the chaotic character was found, and the nonlinear parameter and characteristic values of chaos theory were calculated to analyze the nonlinearity of specimens. By comparisons, the conclusion could be found that the Lyapunov exponent was sensitive to weaker nonlinearity and unsusceptible to noise.
\end{abstract}

Keywords: nonlinear ultrasonic; nondestructive testing; chaos; Lyapunov exponent.

\section{Introduction}

The nonlinear interaction would occur when two kinds of ultrasonic waves propagated through the medium owing to the nonlinearity of tested samples, the waveform had distorted and the energy had redistributed, there were new frequencies in addition to the driving frequency [1].

Ultrasonic nonlinear modulation signal picked up by receiving sensor in nonlinear ultrasonic testing system was nonlinear time series which reflected the cumulative damage of specimen and was complicated, even chaotic in time-domain [2,3]. Because of nonlinear time series was a presentation of nonlinear system, relevant features of system could be gotten by analyzing them by using the chaos and fractal theory. The expansion of fatigue cracks was a study of process and evolution, which belongs to the category of chaos theory, therefore the chaos theory could be used to analyze the modulation signal in principle and the character of fatigue cracks should be obtained [4].

Due to fatigue experiment of metal was time-consuming and expensive, in order to avoid waste of resource and time, therefore in this paper according to the character of ultrasonic nonlinear modulation signal, the simulation signal of it was built, the frequency-domain method and chaos theory were used to analyze the simulation signals, the nonlinear parameter and characteristic values of chaos were calculated to evaluate the nonlinearity of specimen, and the effectiveness of chaos and fractal theory to analyze the modulation signal of receiver was theoretically verified.

\footnotetext{
${ }^{\mathrm{a}}$ Corresponding author : zhangyuhua0422@163.com
} 


\section{The simulation signal of ultrasonic nonlinear modulation effect}

\subsection{Ultrasonic nonlinear modulation effect}

when two kinds of sinusoidal ultrasonic waves propagated in the medium, if there aren't defects in medium, their frequency are unchanged and amplitudes conform to the linear superposition principle; while there are micro cracks or damage defects, it would occur nonlinear interaction and new frequencies $f_{1} \pm f_{2}$ appear in addition to the driving frequencies, as shown in Figure $1[5,6]$.

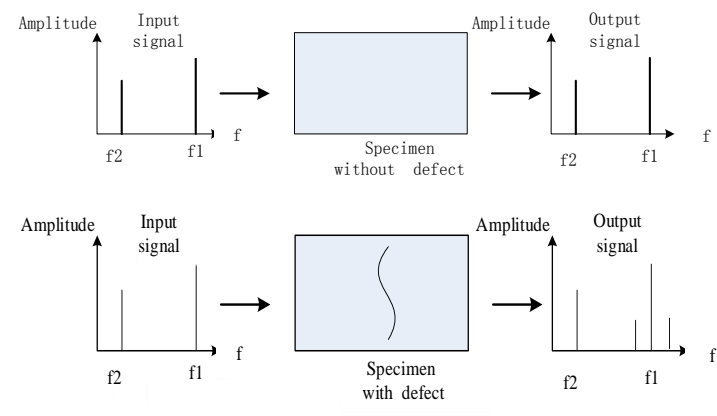

Figure 1. Ultrasonic nonlinear modulation effect

\subsection{The simulation signal of ultrasonic nonlinear modulation effect}

The nonlinear modulation signal was non-sinusoidal time series which reflected cumulative damage of specimens [8]. Due to the effectiveness of chaos theory to analyze the modulation signal should be theoretically verified, nonlinear time series was used to construct and simulate the modulation signal based on transmission character of ultrasonic. Depending on whether consideration ultrasonic attenuation, two cases of nonlinear time series were constructed.

\subsubsection{Without ultrasonic attenuation}

In wave mixing measurement system, two tone bursts of $3 \mathrm{MHz}$ and $10 \mathrm{MHz}$ were fed into power amplifier and low-pass filter, where $f_{1}$ was $10 \mathrm{MHz}$, then the signal of receiver was non-sinusoidal time series after modulation effect.

When constructing the nonlinear time series, two exciting frequencies were $3 \mathrm{MHz}$ and $10 \mathrm{MHz}$, so the excitation signal was $x_{0}=A_{1} \sin \left(2 \pi f_{1} t\right)+A_{2} \sin \left(2 \pi f_{2} t\right)$. For ease of calculating the nonlinear parameter, amplitudes of excitation signals were set as $A_{1}=A_{2}=100 \mathrm{~V}$. Without considering ultrasonic attenuation, amplitudes of sidebands $A_{3}=A\left(f_{1}-f_{2}\right)$ and $A_{4}=A\left(f_{1}+f_{2}\right)$ were the same. Because the modulation signal contained sideband frequencies, the simulation signal was constructed as follows,

$$
\begin{aligned}
& f_{1}=1 \times 10^{7}, f_{2}=3 \times 10^{6}, f_{3}=f_{1}-f_{2}, f_{4}=f_{1}+f_{2} ; \\
& x_{0}=100 \sin \left(2 \pi f_{1} t\right)+50 \sin \left(2 \pi f_{2} t\right) ; \\
& x^{\prime}=100 \sin \left(2 \pi f_{1} t\right)+50 \sin \left(2 \pi f_{2} t\right)+A_{3} \sin \left(2 \pi f_{3} \pi t\right)+A_{4} \sin \left(2 \pi f_{4} t\right)
\end{aligned}
$$

In order to explore changes trend of characteristic values of chaos when the nonlinear effect strengthened [7], the simulation signal $x$ was constructed with increasing amplitudes of $A_{3}$ and $A_{4}$ from $0.05 \mathrm{~V}, 0.1 \mathrm{~V}, 0.15 \mathrm{~V}, 0.2 \mathrm{~V}, 0.25 \mathrm{~V}, 0.5 \mathrm{~V}, 0.75 \mathrm{~V}$, in turn to $5 \mathrm{~V}$, this represented the fatigue cycles of specimen increasing from $5 \%$ to $60 \%$ according to Yan bingsheng's research [9]. 
In order to avoid multiple reflection and refraction of ultrasonic which the modulation signal was more complex should be avoided, 4096 function points of excitation signal were selected when the sampling frequency was $50 \mathrm{MHz}$. Because of the modulation signal inevitably would be affected by interference, the random noise was added to simulation signal which expectation was $a$ and standard deviation was $b$, and the influence of noise was changed by adjusting values of $a$ and $b$.

\subsubsection{With ultrasonic attenuation}

In the process of transmission, the energy gradually weakened due to the diffusion of wave. When the speed of ultrasonic $c$ was stable, the propagation distance was proportional to time, hence ultrasonic attenuation increased with propagation time. When constructing the simulation signal $x^{\prime}$ of modulation effect with ultrasonic attenuation, $\alpha_{1}, \alpha_{2}, \alpha_{3}$ and $\alpha_{4}$ were attenuation coefficient of frequency $f_{1}, f_{2}, f_{3}$ and $f_{4}$ in the solid medium [7].

$$
\begin{aligned}
& f_{1}=1 \times 10^{7}, f_{2}=3 \times 10^{6}, f_{3}=f_{1}-f_{2}, f_{4}=f_{1}+f_{2} ; \\
& x_{0}=100 \sin \left(2 \pi f_{1} t\right)+50 \sin \left(2 \pi f_{2} t\right) ; \\
& x^{\prime}=100 \sin \left(2 \pi f_{1} t\right) e^{-\alpha_{1} c t}+50 \sin \left(2 \pi f_{2} t\right) e^{-\alpha_{2} c t}+A_{3} \sin \left(2 \pi f_{3} \pi t\right) e^{-\alpha_{3} c t}+A_{4} \sin \left(2 \pi f_{4} t\right) e^{-\alpha_{4} c t}
\end{aligned}
$$

When analyzing the simulation signal, 4096 function points were selected, and the random noise was added to veritably simulate the modulation signal.

\section{Chaos theory and characteristic values}

Based on the simulation signals of modulation effect, the frequency-domain method and chaos theory were used to analyze them, the nonlinear parameter and characteristic values of chaos were calculated, therefore chaos theory and characteristic values were introduced in this section.

In time-domain analysis, the nonlinear time series $x_{1}, x_{2}, x_{3} \ldots x_{\mathrm{n}} \ldots$ displays confusing and complex characteristics. The phase space reconstruction method uses appropriate embedding dimension $m$ and time delay $\tau$ to reconstitute $\mathrm{m}$-dimensional space and restore dynamic properties of attractor [2].

Characteristic values describe features of attractor, for example the Lyapunov exponent depicts the separation rate of phase space trajectories, K entropy describes complex degree of system, while correlation dimension measures the complexity of attractor in phase space [3]. In the process of fatigue crack extension, the size, quantity, and complexity of the extension path are changing with the cyclic loading, so the variation of Lyapunov exponent, $\mathrm{K}$ entropy and correlation dimension could describe the process [10].

\section{Results and discussion}

\subsection{Chaotic characteristics of simulation signal $x$}

Based on the simulation signal $x$, variation curves of the nonlinear parameter of $f_{3}$ and $f_{4}$, the Lyapunov exponent, correlation dimension, K entropy were shown in Figure2. There are seven curves with different marking in each coordinate system which represent the simulation signal $x$ with different signal-to-noise ratio, this can analyze the influence of noise to characteristic values. 

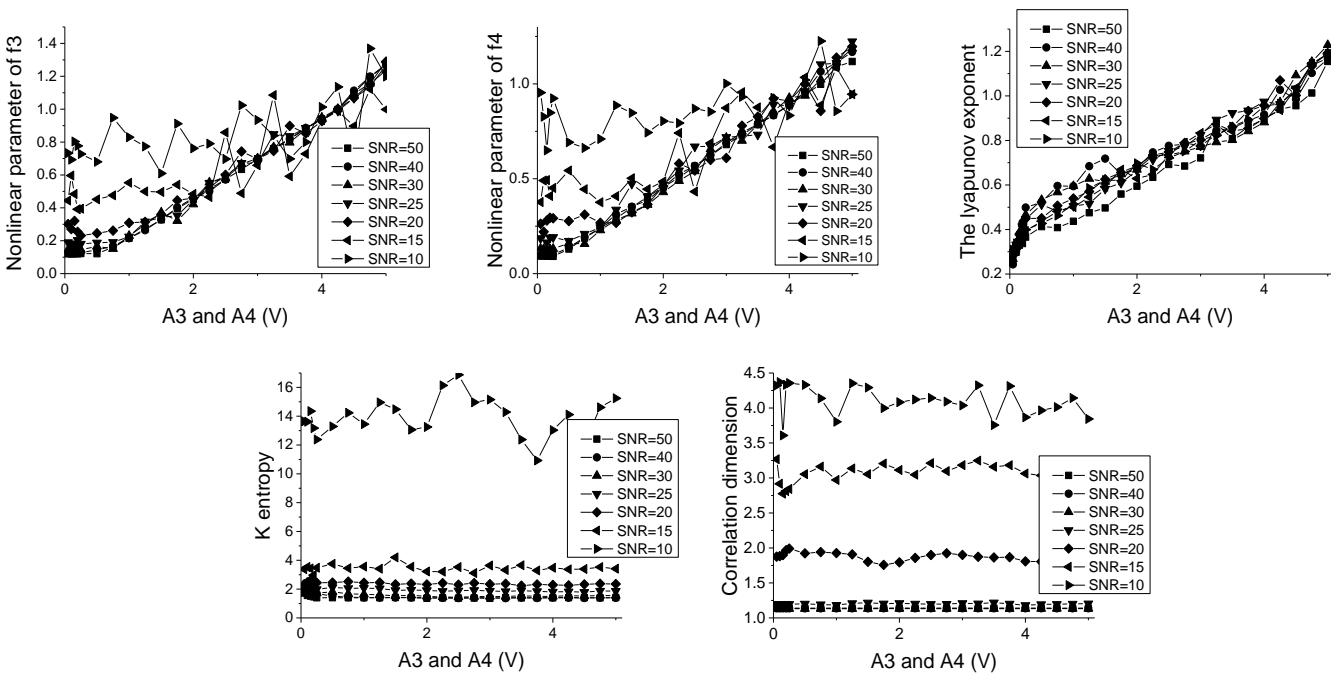

Figure 2. Variation curves of characteristic values without ultrasonic attenuation

Firstly variation curves of the nonlinear parameter of $f_{3}$ and $f_{4}$ were analyzed. Curves were almost flat with $\mathrm{x}$-axis at weaker nonlinearity, this meant that the parameter couldn't properly characterize the nonlinearity enhancing. When $A_{3}$ and $A_{4}$ were greater than $0.75 \mathrm{~V}$, the nonlinear parameter monotonically increased. With the loss of signal-to-noise ratio, the volatility of curves was bigger and bigger; this explained the nonlinear parameter couldn't represent nonlinearity at strong interference.

That the lyapunov value were greater than 0.2 with increase of nonlinearity illustrated the simulation signal had chaotic character. With the loss of SNR, that variation curves of the Lyapunov exponent were consistent showed the noise of simulation signal had little influence on the characteristic value. The Lyapunov exponent monotonously fortified with increase of $A_{3}$ and $A_{4}$, especially when amplitudes were smaller than $0.5 \mathrm{~V}$, the curves were much steeper, this indicated the Lyapunov exponent could properly represent the nonlinearity of material and was more sensitive to weaker nonlinearity.

Then analysis variation curves of $\mathrm{K}$ entropy and correlation dimension together because their changing trend were the same. Due to K entropy and correlation dimension added about 2\% and 3.5\% respectively with increase of $A_{3}$ and $A_{4}$, the curve seemed flat with the x-axis because the increasing range was relatively small comparing with the range of y-axis. With decrease of signal-to-noise ratio the distortion degree and confusion extent of nonlinear time series increased in exponential way especially when the SNR was below 20. Since K entropy measured the randomness of system and correlation dimension measured the complexity of attractor, therefore the $\mathrm{K}$ entropy and correlation dimension fortified quickly along the y-axis in the coordinate system.

\subsection{Chaotic characteristics of simulation signal $x^{\prime}$}

The variation of characteristic values of signal $x^{\prime}$ were shown in Fig.3. 

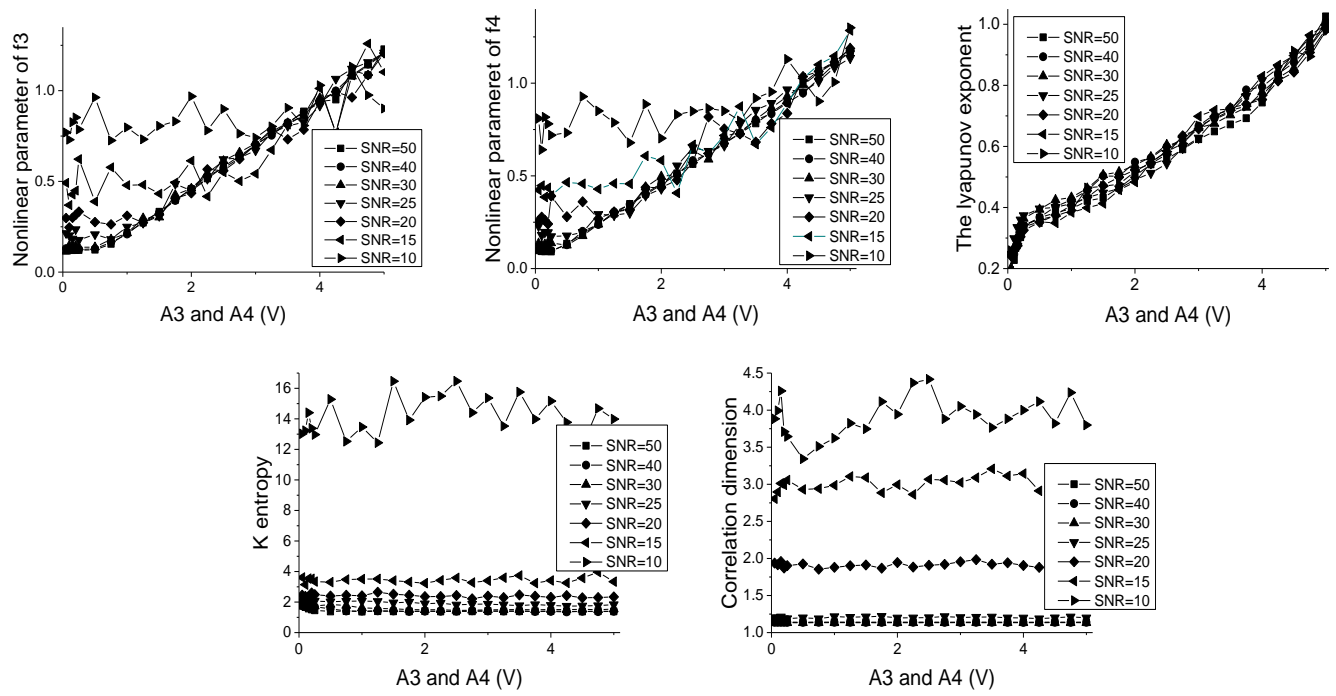

Figure 3. The variation curve of characteristic values with ultrasonic attenuation

Contrast analysis Figure 2 and Figure 3, the variation tendency of characteristic values was similar in two kinds of simulation signals. Ultrasonic attenuation had little influence on the nonlinear parameter, the Lyapunov exponent, correlation dimension and K entropy.

Studies had shown that the nonlinearity of material mainly resulted from dislocation slip, microcracks and other microscopic defects [11]. With action of alternating load, the stress concentration zone of specimens would appear crack nucleation which was the earlier stage of performance degradation and the nonlinearity was very weaker. When amplitudes of sidebands were small, the nonlinear parameter wasn't sensitive to weaker nonlinearity, while the Lyapunov exponent increased rapidly indicating that it was sensitive to early performance degradation. The volatility of the nonlinear parameter were bigger and bigger with the loss of SNR while the variation trend of Lyapunov exponent was consistent, The analysis showed the nonlinear parameter was susceptible to noise while the lyapunov exponent was insensitive to interference of time series.

\section{Conclusion}

The chaos and fractal theory was firstly introduced in nonlinear ultrasonic technique. The conclusions of this paper are:

(1)Two kinds of simulation signals with increasing amplitudes of sidebands which represented the nonlinearity of specimen increasing were built in wave mixing method whether consideration ultrasonic attenuation.

(2) Simulation signals had chaotic character by calculating the biggest lyapunov exponent.

(3) The variation of the nonlinear parameter and chaotic characteristics were gotten based on simulation signals. The nonlinear parameter was susceptible to interference and not sensitive to weaker nonlinearity, while the Lyapunov exponent was impressible to weaker nonlinearity and unsusceptible to noise. Therefore the effectiveness of chaos and fractal theory in analyzing ultrasonic nonlinear modulation signal was theoretically verified. So a new analysis and feature extraction method for nonlinear ultrasonic testing was provided

\section{Acknowledgments}

This work was supported by the National Natural Science Foundation of China (grant nos. 51445013 and 51365006). 


\section{Reference}

1. G. S. Shui, P. Huang, and Y. S. Wang, Non-destructive Evaluation of Fatigue Damage of Train Spring using Nonlinear Ultrasonic Method, Acta Acoustics. 5, 570(2013).

2. Lv, J. H., J. A. Lu, and S. H. Chen, Chaotic Time Series Analysis and Application (Wuhan university press, Wuhan, 2002), (In Chinese).

3. X. Li, T. L. Li, H. Y. Quan, and X. Tian, Analysis of EHRV based on Correlation Dimension and largest Lyapunov Exponent, Journal of Biomedical Engineering Research. 3, 188(2009).

4. K. Holger, and S. Thomas, Nonlinear Time Series Analysis (Cambridge university press, Britain, 2004).

5. Z. G. Zhou, and S. M. Liu. Nonlinear Ultrasonic Techniques Used in Nondestructive Testing: A Review, Journal of Mechanical Engineering. 8, 2(2011), (In Chinese).

6. J. P. Jiao, J. J. Sun, G. H. Li, B. Wu, and C. F. He, Evaluation of the Inter granular Corrosion in Austenitic Stainless Steel using Collinear Wave Mixing Method, NDT \& E International. 1(2015).

7. H. F. Hu, Research on Theory and key Technologies of Nonlinear Ultrasonic for Health Monitoring of Plate-like Structures, D.E. thesis, National University of Defense Technology, (Changsha, China, 2011), (In Chinese).

8. Z. H. Chen, Y. W. Shi, and H. Y. Zhao, Nonlinear Ultrasonic Testing and Imaging for tiny Flaw, Acta Acoustics. 8, 9(2010).

9. B. S. Yan, Z. R. Liu, Y. C. Zhang, and B. P. Tang, Experimental Study of Early Fatigue Nonlinear Ultrasonic Detection in Magnesium Alloy, Journal of Mechanical Engineering. 4, 20(2013), (In Chinese).

10. F. Liu, Vibration Fault Mode Determination for Rotor based on Correlation Dimension and Kolmogorov Entropy, Master's thesis, Shenyang institute of aeronautical engineering, (Shenyang, China, 2009), (In Chinese).

11. M. Liao, Dislocation Theory based Short Crack Model and its Application for Aircraft Aluminum Alloys, Engineering Fracture Mechanics, 1, 22(2010). 